\title{
Iterative Learning Control with Extended State Observer for Telescope System
}

\author{
Huaxiang Cai, ${ }^{1,2,3}$ Yongmei Huang, ${ }^{1,2}$ Junfeng Du, \\ Tao Tang, ${ }^{1,2}$ Dan Zuo, ${ }^{1,2,3}$ and Jinying $\mathrm{Li}^{\mathbf{1}, 2}$ \\ ${ }^{1}$ Chinese Academy of Sciences Institute of Optics and Electronics, P.O. Box 350, Shuangliu, Chengdu 610209, China \\ ${ }^{2}$ Key Laboratory of Optical Engineering, Chinese Academy of Sciences, Chengdu, China \\ ${ }^{3}$ University of Chinese Academy of Sciences, Beijing 100039, China \\ Correspondence should be addressed to Huaxiang Cai; chx_ioe@163.com
}

Received 4 March 2015; Revised 24 June 2015; Accepted 1 July 2015

Academic Editor: Xinggang Yan

Copyright (C) 2015 Huaxiang Cai et al. This is an open access article distributed under the Creative Commons Attribution License, which permits unrestricted use, distribution, and reproduction in any medium, provided the original work is properly cited.

\begin{abstract}
An Iterative Learning Control (ILC) method with Extended State Observer (ESO) is proposed to enhance the tracking precision of telescope. Telescope systems usually suffer some uncertain nonlinear disturbances, such as nonlinear friction and unknown disturbances. Thereby, to ensure the tracking precision, the ESO which can estimate system states (including parts of uncertain nonlinear disturbances) is introduced. The nonlinear system is converted to an approximate linear system by making use of the ESO. Besides, to make further improvement on the tracking precision, we make use of the ILC method which can find an ideal control signal by the process of iterative learning. Furthermore, this control method theoretically guarantees a prescribed tracking performance and final tracking accuracy. Finally, a few comparative experimental results show that the proposed control method has excellent performance for reducing the tracking error of telescope system.
\end{abstract}

\section{Introduction}

A high performance telescope system has attracted lots of attention, since it is widely used in space applications, such as space observer [1] and satellite surveillance [2]. However, it is inevitable that there are always some nonlinear characteristics in the telescope tracking control systems, such as deadzone [3], friction [4], and some uncertainly disturbances [5]. Without doubt, all the nonlinear features will deteriorate the tracking performance of systems. Simultaneously, the conventional linear control method (proportional-integral) has not guaranteed a higher tracking precision. To solve the above nonlinear problems, there are many control methods being proposed, such as adaptive control [6], slide control [7], compensating control based on DOB [8], active disturbance rejection control (ADRC) [9], and iterative learning control (ILC) [10].

In recent years, since the iterative learning control (ILC) needs too little knowledge of system dynamics, it has received a great deal of attention [11]. ILC is proposed by Arimoto et al. in 1984 [12]. It is essentially a feedforward control approach that fully utilizes the previous control information [13]. Over the past three decades, it has been successfully used in extensive research fields such as industrial robotics [14], manufacturing process [15], stochastic process control system [16], and hysteretic system [17]. Nevertheless, the ILC still has some of its inherent shortages. It can only eliminate some disturbances which emerge repeatedly. If there are some nonlinear nonperiod disturbances involved in the systems, the sole ILC method will face difficulty to get a higher tracking precision. The ADRC is different from the ILC, which can estimate unknown nonlinear disturbances involved in the systems. As the core technology of ADRC, the ESO also needs to know little knowledge of system dynamics, which only needs the information of system's input and output. Thereby, it has been widely used in many fields such as missile control system [18], optical system [19], and other high precision occasions [20]. Even though the ESO has excellent estimation ability for system states, its estimation ability is still restricted to the bandwidth of ESO. 
In this paper, since there are some uncertain nonlinear disturbances in the telescope system, which reduce severely the tracking precision of system, it is needed to eliminate the nonlinear disturbances and find the best input signal for the system. Under the circumstances, an ILC technique with ESO is proposed, which takes use of the advantages of the two control methods (ILC and ESO). The function of ESO is to eliminate parts of uncertain nonlinear disturbances and to transform the nonlinear system into an approximate linear system. And the function of ILC is to get the best input signal for the approximate linear system. In the ILC controller, a PD-type learning algorithm with the forgetting factor is chosen. The forgetting factor can balance the learning precision and the robustness of system. To illustrate the excellent performance of the proposed control method, three comparative experiments will be given in this paper.

This remainder of this paper is organized as follows. Section 2 gets the dynamic models. Section 3 introduces the controller design and the theory analysis. The experiment results are presented in Section 4. And some conclusions can be found in Section 5 .

\section{Dynamic Models}

The tracking control system is made of brushless DC, load, high precision encoder, servo actuator, and host computer. The goal is to make the system track the given reference motion trajectory as far as possible. The control structure is shown in Figure 1.

In the telescope system, since the electrical response of the actuator is very fast, the current dynamics can be neglected. And the dynamics of the tracking system is

$$
\begin{aligned}
& \frac{d \theta(t)}{d t}=\omega(t) ; \\
& \frac{d \omega(t)}{d t}=\frac{u}{J}-\frac{v}{J} \omega(t)-f(t, \omega) ;
\end{aligned}
$$

$$
u=k_{t} i, J=J_{m}+J_{l} .
$$

In (1), $J_{m}$ and $J_{l}$ are the motor inertia and the load inertia, respectively. $\theta$ and $\omega$ represent for the load angle position and the load angle velocity, respectively. $u$ represents the torque imposed on the motor. $i$ is the control input current of the motor. $f(t, \omega)$ represents nonlinear disturbances (such as the friction disturbance, external disturbances, and some unmodeled dynamics). $v$ is the viscous coefficient.

Rewrite the dynamic model (1) as

$$
\begin{aligned}
& \frac{d \theta(t)}{d t}=\omega(t) \\
& \frac{d \omega(t)}{d t}=b_{0} u+f_{1}(t, \omega) ;
\end{aligned}
$$

$$
u=k_{t} i, J=J_{m}+J_{l}
$$

$f_{1}(t, \omega)=\left(1 / J-b_{0}\right) u-(\nu / J) \omega(t)-f(t, \omega)$, which includes the nonlinear disturbances $-f(t, \omega)$ and the inner disturbances $\left(1 / J-b_{0}\right) u-(\nu / J) \omega(t)$. Here, $b_{0}$ is a parameter that can be adjusted in the controller.

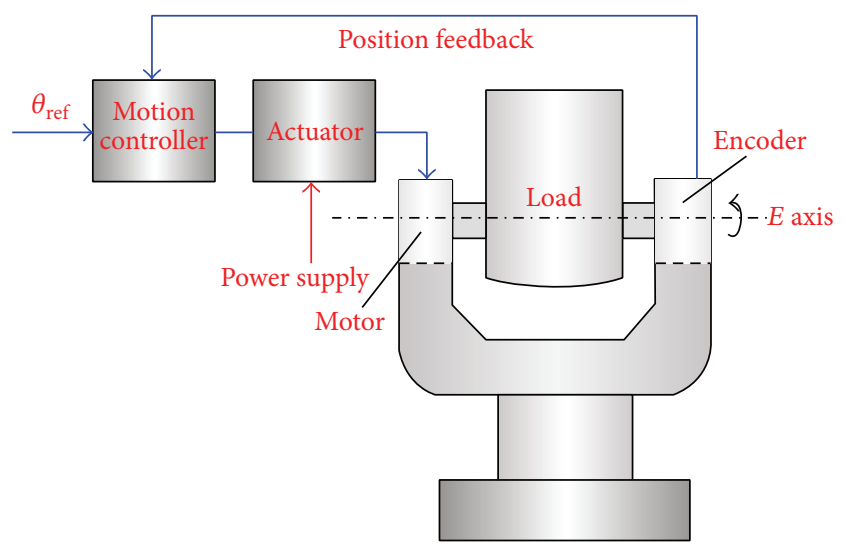

FIGURE 1: Architecture of tracking system.

\section{Controller Design}

The aim of designing controller is eliminating the nonlinear disturbance $f_{1}(t, \omega)$ and enhancing the tracking precision of system. The ESO makes the nonlinear system transform into an approximate linear system. And the ILC with ESO gets the ideal input control signal.

In most of telescope systems, the multiple-loops control structure is usually applied to guarantee the tracking precision of systems, which includes position loop, velocity loop, and current loop. In this paper, since the current dynamic has higher frequency range than other loops, it has been ignored. We mainly analyze position loop and speed loop.

\subsection{Design PI Controller with ESO}

3.1.1. Design the ESO. Before designing the ESO, we assume that the disturbance $f_{1}(t, \omega)$ is continuous and differential.

Assumption 1. The disturbance $f_{1}(t, \omega)$ is continuous and differential, and $h=-d f_{1}(t, \omega) / d t$. Besides, $h(t)$ is bounded; namely, there is a positive constant $\beta$ to meet:

$$
\beta=\sup \{t>0|h(t)|\} .
$$

Consequently, we extend the $f_{1}(t, \omega)$ as a separate state $x_{3}$ of system. Then, the original plant of system (2) can be described as

$$
\begin{aligned}
& \dot{x}_{1}(t)=x_{2}(t) ; \\
& \dot{x}_{2}(t)=x_{3}(t)+b_{0} u(t) ; \\
& \dot{x}_{3}(t)=-h,
\end{aligned}
$$

where $x=\left[\begin{array}{lll}x_{1} & x_{2} & x_{3}\end{array}\right]^{T}=\left[\begin{array}{lll}\theta & \omega & f_{1}\end{array}\right]^{T}$.

By (4), we can know that it is observable. Hence, an ESO can be constructed as (5)

$$
\begin{aligned}
& \dot{\hat{x}}_{1}(t)=\widehat{x}_{2}(t)-\beta_{01}\left[\widehat{x}_{1}(t)-x_{1}(t)\right] \\
& \dot{\hat{x}}_{2}(t)=\widehat{x}_{3}(t)-\beta_{02}\left[\widehat{x}_{1}(t)-x_{1}(t)\right]+b_{0} u(t) ; \\
& \dot{\hat{x}}_{3}(t)=-\beta_{03}\left[\widehat{x}_{1}(t)-x_{1}(t)\right]
\end{aligned}
$$


where $\beta_{01}, \beta_{02}$, and $\beta_{03}$ are the parameters that will be designed in the ESO.

The aim of ESO is to make

$$
\begin{aligned}
& \widehat{x}_{1}(t) \longrightarrow x_{1}(t), \\
& \widehat{x}_{2}(t) \longrightarrow x_{2}(t), \\
& \widehat{x}_{3}(t) \longrightarrow f_{1}(t, \omega) .
\end{aligned}
$$

Let $\eta_{i}=\widehat{x}_{i}-x_{i}, i=1,2,3$, denote the estimation error; subtracting (4) from (5), then we can obtain

$$
\dot{\eta}=A \eta+M h
$$

where

$$
\begin{aligned}
\eta & =\left[\begin{array}{l}
\eta_{1} \\
\eta_{2} \\
\eta_{3}
\end{array}\right], \\
A & =\left[\begin{array}{lll}
-\beta_{01} & 1 & 0 \\
-\beta_{02} & 0 & 1 \\
-\beta_{03} & 0 & 0
\end{array}\right], \\
M & =\left[\begin{array}{l}
0 \\
0 \\
1
\end{array}\right] .
\end{aligned}
$$

Therefore, if the matrix $A$ is Hurwitz, the ESO will be Bounded-Input Bounded-Output (BIBO) stable. In the matrix $A$, the parameters $\beta_{01}, \beta_{02}$, and $\beta_{03}$ can be designed as [21]

$$
\begin{aligned}
& \beta_{01}=3 \omega_{0}, \\
& \beta_{02}=3 \omega_{0}^{2}, \\
& \beta_{03}=\omega_{0}^{3},
\end{aligned}
$$

where $\omega_{0}$ is the bandwidth of ESO. Equation (9) can promise the matrix $A$ being Hurwitz.

Lemma 2. If the $h(t)$ is bounded, there will be a positive constant $\zeta_{i}>0$ and a finite time $T_{1}>0$ such that

$$
\begin{aligned}
\left|\eta_{i}(t)\right| & \leq \zeta_{i}, \\
\zeta_{i} & =\mathrm{o}\left(\frac{1}{\omega_{0}^{c}}\right) ;
\end{aligned}
$$

$$
i=1,2,3 \forall t \geq T_{1} .
$$

The proof can be found in the literature [22].

Remark 3. Equation (9) makes the ESO (5) a BIBO stable observer. The result of Lemma 2 shows that the ESO has an excellent estimation ability for disturbances. Its estimation ability depends on the bandwidth $\omega_{0}$ of ESO. When the bandwidth $\omega_{0}$ is big enough, it can estimate accurately $x_{1}(t)$, $x_{2}(t)$, and $f_{1}(t, \omega)$.
According to the analysis in Lemma 2, when designing an appropriate parameter $\omega_{0}$, it can make $o\left(1 / \omega_{0}^{c}\right) \approx 0$. And there will be $\left|x_{3}-\widehat{x}_{3}\right|=\left|\eta_{3}(t)\right| \leq o\left(1 / \omega_{0}^{c}\right)$; namely, the $\widehat{x}_{3} \approx f_{1}(t, \omega)$. So we can transform the input $u$ into $u_{\omega}$; namely, $u=\left(u_{\omega}-\right.$ $\left.\widehat{x}_{3}\right) / b_{0}$.

Substituting the $u=\left(u_{\omega}-\widehat{x}_{3}\right) / b_{0}$ to (2), we can get

$$
\begin{aligned}
& \frac{d \theta(t)}{d t}=\omega(t) ; \\
& \frac{d \omega(t)}{d t} \approx u_{\omega} .
\end{aligned}
$$

3.1.2. Design Velocity Loop PI Controller. The nonlinear system (2) is converted to an approximate linear integral system (11). So we can design a PI controller in the velocity loop:

$$
u_{\omega}=K_{p}^{\omega}\left(\omega^{\mathrm{ref}}-\widehat{x}_{2}\right)+K_{i}^{\omega} \int_{0}^{t}\left[\omega^{\mathrm{ref}}(\tau)-\widehat{x}_{2}(\tau)\right] d \tau,
$$

where $\omega^{\text {ref }}$ is the input signal of velocity loop and $K_{p}^{\omega}, K_{i}^{\omega}$ are the proportion gain and the integral gain in the velocity loop controller, respectively.

3.1.3. Design Position Loop PI Controller. Similarly, we can design a PI controller in the position loop:

$$
\omega^{\mathrm{ref}}=K_{p}^{\theta}\left(\theta^{\mathrm{ref}}-\widehat{x}_{1}\right)+K_{i}^{\theta} \int_{0}^{t}\left[\theta^{\mathrm{ref}}(\tau)-\widehat{x}_{1}(\tau)\right] d \tau,
$$

where $\theta^{\text {ref }}$ is the input signal of position loop and $K_{p}^{\omega}, K_{i}^{\omega}$ are the proportion gain and the integral gain in the position loop controller, respectively.

3.2. Iterative Learning Controller with ESO Design. From (11), we can see that the system is only an approximate linear system. Thereby, to make further improvement on the tracking precision of system, we will find an ideal input $u_{\omega}$ by the method of iterative learning. And it can make the output $\omega$ of system close to the input $\omega^{\text {ref }}$ as far as possible. In this section, we will design an ILC to substitute the PI controller in the velocity loop.

According to (11), the state space equation of system can be obtained as

$$
\begin{aligned}
\dot{x}(t) & =A_{l} x+B u_{\omega} ; \\
y & =C x,
\end{aligned}
$$

where $x=\left[\begin{array}{l}x_{1} \\ x_{2}\end{array}\right]=\left[\begin{array}{l}\theta \\ \omega\end{array}\right], A_{l}=\left[\begin{array}{ll}0 & 1 \\ 0 & 0\end{array}\right], B=\left[\begin{array}{l}0 \\ 1\end{array}\right]$, and $C=\left[\begin{array}{ll}0 & 1\end{array}\right]$.

To make the system track the desired reference trajectory, we assume that there is an ideal input signal meeting the condition.

Assumption 4. The ideal input signal $u_{d}(t)$ can make the system track the desired reference trajectory $y_{d}(t)$ :

$$
\begin{aligned}
& \dot{x}_{d}(t)=A_{l} x_{d}(t)+B u_{d}(t) ; \\
& y_{d}(t)=C x_{d}(t),
\end{aligned}
$$

where $x_{d}(t), y_{d}(t)$ represent the ideal states of system, respectively. 


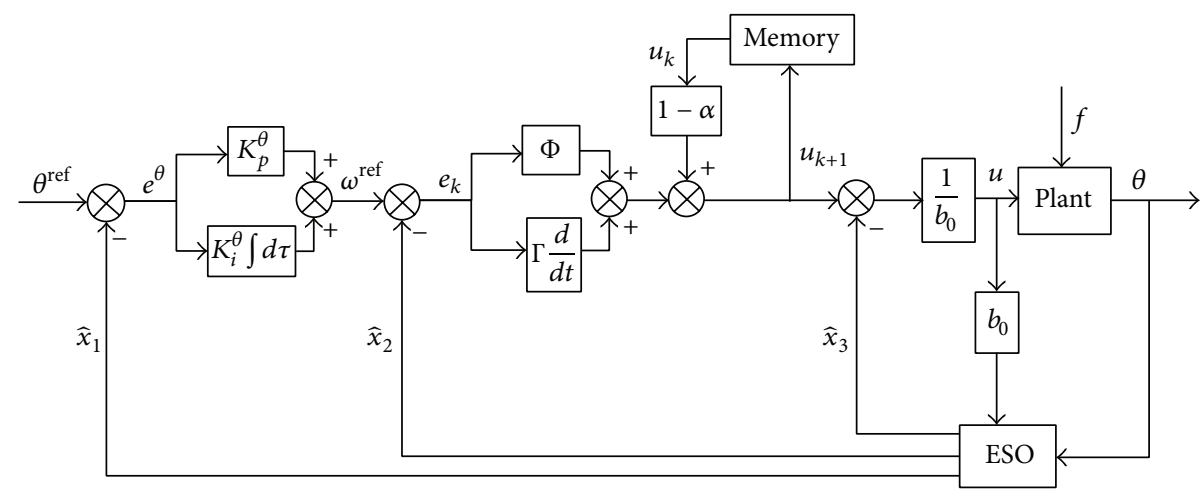

FIGURE 2: Schematic diagram of the proposed iterative learning control with ESO.

Since system (14) will be tracked a period trajectory, there will be once input in every period. Then the state space equation of system (14) can be rewritten:

$$
\begin{aligned}
\dot{x}_{k}(t) & =A_{l} x_{k}+B u_{k} ; \\
y_{k} & =C x_{k},
\end{aligned}
$$

where $k$ is the times. Equation (16) means the $k$ th states of system under the $k$ th input $u_{k}$.

For system (16), a PD-type learning algorithm with the forgetting factor $\alpha$ is selected. The factor $\alpha$ is introduced to make a tradeoff between perfect learning and robustness, which can increase the robustness of ILC against noise, initialization error and fluctuation of system dynamics [23]. The selected iterative learning scheme is found in (17), whose schematic diagram is depicted in Figure 2:

$$
\begin{aligned}
u_{k+1}(t)= & (1-\alpha) u_{k}(t)+\alpha u_{0}(t)+\Phi\left(e_{k}(t)\right) \\
& +\Gamma\left(\dot{e}_{k}(t)\right)
\end{aligned}
$$

where $\Phi$ and $\Gamma$ are the proportion gain matrix and deviation gain matrix, respectively. $\Phi \in \mathbb{R}^{m \times q}, \Gamma \in \mathbb{R}^{m \times q}$ are bounded. $k$ is the times. $e_{k}(t)=y_{d}(t)-y_{k}(t)$ is the iterative error in the $k$ th times.

\subsection{Iterative Learning Controller Convergence Analysis}

Theorem 5. If the system described by (16) satisfies assumptions and uses the update law (18). Given a desired trajectory $y_{d}(t)$ and an initial state $x_{d}(0)$ which are achievable, if

$$
\|(1-\alpha) I-\Gamma C B\| \leq \rho<1,
$$

then, as $k \rightarrow \infty$, the iterative output $y_{k}(t)$ of system will converge to the desired reference trajectory $y_{d}(t)$.
Proof. Consider

$$
\begin{aligned}
u_{d}(t)-u_{k+1}(t)= & u_{d}(t)-(1-\alpha) u_{k}(t)-\alpha u_{0}(t) \\
& -\Phi\left(e_{k}(t)\right)-\Gamma\left(\frac{d\left(e_{k}(t)\right)}{d t}\right) \\
= & (1-\alpha)\left[u_{d}(t)-u_{k}(t)\right] \\
& +\alpha\left[u_{d}(t)-u_{0}(t)\right] \\
& -\Phi C\left[x_{d}(t)-x_{k}(t)\right] \\
& -\Gamma C A\left[x_{d}(t)-x_{k}(t)\right] \\
& -\Gamma C B\left[u_{d}(t)-u_{k}(t)\right] \\
= & {[(1-\alpha) I-\Gamma C B]\left[u_{d}(t)-u_{k}(t)\right] } \\
& -(\Phi C+\Gamma C A)\left[x_{d}(t)-x_{k}(t)\right] .
\end{aligned}
$$

In (19), by the state space equation (15) and (16), the $x_{d}(t)-x_{k}(t)$ can be transformed into

$$
\begin{aligned}
x_{d}(t)-x_{k}(t)= & e^{A t}\left[x_{d}(0)-x_{k}(0)\right] \\
& +\int_{0}^{t} e^{A(t-\tau)} B\left[u_{d}(\tau)-u_{k}(\tau)\right] d \tau .
\end{aligned}
$$

Substituting (20) to (19), we can have

$$
\begin{aligned}
u_{d}(t) & -u_{k+1}(t) \\
= & {[(1-\alpha) I-\Gamma C B]\left[u_{d}(t)-u_{k}(t)\right] } \\
& -(\Phi C+\Gamma C A) e^{A t}\left[x_{d}(0)-x_{k}(0)\right] \\
& -(\Phi C+\Gamma C A) \int_{0}^{t} e^{A(t-\tau)} B\left[u_{d}(\tau)-u_{k}(\tau)\right] d \tau .
\end{aligned}
$$


Taking the norm $\|\cdot\|$ on both sides of (21), we obtain

$$
\begin{aligned}
&\left\|u_{d}(t)-u_{k+1}(t)\right\| \\
& \leq\|(1-\alpha) I-\Gamma C B\| \cdot\left\|u_{d}(t)-u_{k}(t)\right\| \\
&+e^{A t}\|\Phi C+\Gamma C A\| \cdot\left\|x_{d}(0)-x_{k}(0)\right\| \\
&+\|\Phi C+\Gamma C A\| \\
& \cdot \int_{0}^{t} e^{A(t-\tau)} B\left\|u_{d}(\tau)-u_{k}(\tau)\right\| d \tau .
\end{aligned}
$$

Multiplying by $e^{-\lambda t}, \lambda>\|A\|$, and we have

$$
\begin{gathered}
\left\|u_{d}(t)-u_{k+1}(t)\right\| e^{-\lambda t} \leq\|(1-\alpha) I-\Gamma C B\| \cdot \| u_{d}(t) \\
-u_{k}(t)\left\|e^{-\lambda t}-\right\| \Phi C+\Gamma C A\|\cdot\| x_{d}(0)-x_{k}(0) \| \\
\cdot e^{(\|A\|-\lambda) t}+\|\Phi C+\Gamma C A\| \\
\cdot \int_{0}^{t} e^{(\|A\|-\lambda)(t-\tau)} e^{-\lambda \tau} B\left\|u_{d}(\tau)-u_{k}(\tau)\right\| d \tau \\
\leq\|(1-\alpha) I-\Gamma C B\| \cdot\left\|u_{d}(t)-u_{k}(t)\right\| e^{-\lambda t}-\| \Phi C \\
+\Gamma C A\|\cdot\| x_{d}(0)-x_{k}(0) \| e^{(\|A\|-\lambda) t} \\
+\frac{\|\Phi C+\Gamma C A\| \cdot\|B\| \cdot\left(1-e^{(\|A\|-\lambda) t}\right)}{\lambda-\|A\|} \| u_{d}(t) \\
-u_{k}(t) \| e^{-\lambda t} \cdot
\end{gathered}
$$

Definition 6. Define $\lambda$ norm for a function $h:[0, T] \rightarrow \mathbb{R}^{k}$ [24]; then

$$
\|h(\cdot)\|_{\lambda} \triangleq \sup _{t \in[0, T]} e^{-\lambda t}\|h(\cdot)\| \longrightarrow \mathbb{R}^{k}
$$

Then

$$
\begin{gathered}
\left\|u_{d}(t)-u_{k+1}(t)\right\|_{\lambda} \leq \|(1-\alpha) I-\Gamma C B \\
+\frac{\|\Phi C+\Gamma C A\| \cdot\|B\| \cdot\left(1-e^{(\|A\|-\lambda) t}\right)}{\lambda-\|A\|}\|\cdot\| u_{d}(t) \\
-u_{k}(t)\left\|_{\lambda}-\right\| \Phi C+\Gamma C A\|\cdot\| x_{d}(0)-x_{k}(0) \| .
\end{gathered}
$$

Since the $(1-\alpha) I-\Gamma C B \leq \rho<1$, we can find a $\lambda>\|A\|$ which makes

$$
\begin{aligned}
\bar{\rho}= & (1-\alpha) I-\Gamma C B \\
& +\frac{\|\Phi C+\Gamma C A\| \cdot\|B\| \cdot\left(1-e^{(\|A\|-\lambda) t}\right)}{\lambda-\|A\|} \|<1 .
\end{aligned}
$$

So we can get

$$
\left\|u_{d}(t)-u_{k+1}(t)\right\|_{\lambda} \leq \frac{\|\Phi C+\Gamma C A\|}{1-\bar{\rho}}\left\|x_{d}(0)-x_{k}(0)\right\| \text {. }
$$

Remark 7. It shows that $u_{k}$ converges to the $u_{d}$ of radius $(\|\Phi C+\Gamma C A\| /(1-\bar{\rho}))\left\|x_{d}(0)-x_{k}(0)\right\|$ with respect to the $\lambda$ norm. Besides, we can know that the convergent radius is bounded. And the boundary depends on the initial input states of system.

Consequently, the tracking error of system will be

$$
\begin{gathered}
e_{k+1}(t)=y_{d}(t)-y_{k+1}(t)=C \cdot\left\{e^{A t}\left[x_{d}(0)-x_{k}(0)\right]\right. \\
\left.+\int_{0}^{t} e^{A(t-\tau)} B\left[u_{d}(\tau)-u_{k}(\tau)\right] d \tau\right\}
\end{gathered}
$$

Similarly, taking the norm $\|\cdot\|_{\lambda}$ on both sides of (28), we can get

$$
\begin{aligned}
& \left\|e_{k+1}(t)\right\|_{\lambda}=\left\|y_{d}(t)-y_{k+1}(t)\right\|_{\lambda} \leq\|C\| \\
& \cdot \| e^{A t}\left[x_{d}(0)-x_{k+1}(0)\right] \\
& +\int_{0}^{t} e^{A(t-\tau)} B\left[u_{d}(\tau)-u_{k+1}(\tau)\right] d \tau\left\|_{\lambda} \leq\right\| C \| \\
& \cdot\left\|\left[x_{d}(0)-x_{k+1}(0)\right]\right\| \\
& +\frac{\|C\| \cdot\|B\| \cdot\left(1-e^{(\|A\|-\lambda) t}\right)}{\lambda-\|A\|}\left\|u_{d}(t)-u_{k+1}(t)\right\|_{\lambda} \\
& \leq\|C\| \cdot\left\|\left[x_{d}(0)-x_{k+1}(0)\right]\right\| \\
& +\frac{\|C\| \cdot\|B\| \cdot\left(1-e^{(\|A\|-\lambda) t}\right)}{\lambda-\|A\|} \\
& +\frac{\|\Phi C+\Gamma C A\|}{1-\bar{\rho}}\left\|x_{d}(0)-x_{k}(0)\right\| .
\end{aligned}
$$

Specially, the system usually can meet the initial condition; that is,

$$
x_{d}(0)=x_{k}(0), \quad k=1,2,3, \ldots
$$

That is said as

$$
\lim _{k \rightarrow \infty}\left\|e_{k+1}(t)\right\|_{\lambda} \longrightarrow 0
$$

To sum up, when the PD-type learning algorithm is applied to the ILC with ESO, the input $u_{k}(t)$ of system can converge to the ideal input $u_{d}(t)$, and the output of system can converge to the ideal output $y_{d}(t)$.

\section{Experiment Setup and Result}

The verification platform is the rotary table of telescope system, which consists of a DC motor, a tracking load, an electrical driver, a control system, and a high precision encoder whose accuracy is about \pm 0.618 (arc-second). The control scheme includes two loops: position loop and velocity loop. 


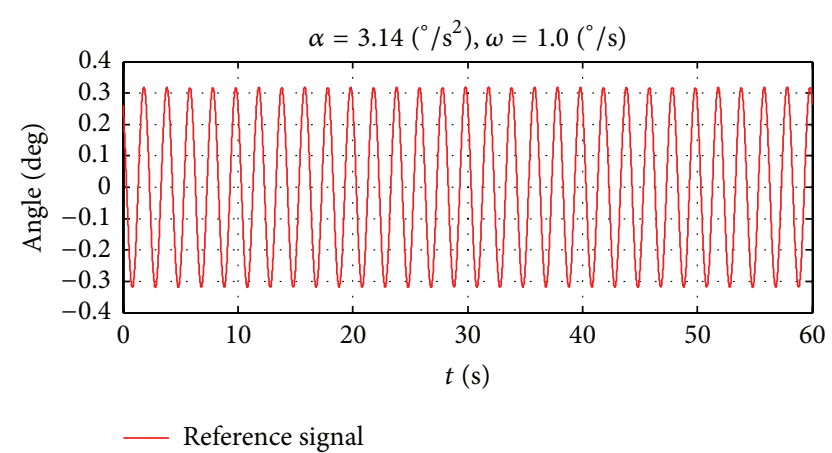

Figure 3: The desired reference signal.

The following three controllers are compared.

(1) PI-PI: this is the traditional Proportional-Integral (PI) controller. In the velocity loop and position loop, we use two PI controllers. The velocity signal can be obtained by differentiating the position signal.

(2) PI-ESO: in both of the position loop and the velocity loop, the PI controllers are used, but the velocity signal will be gotten by the ESO. The bandwidth $\omega_{0}$ of ESO is $\omega_{0}=100$. The parameters of position loop's PI controller remain the same.

(3) PI-ILC-ESO: the PI controller is used in the position loop, and the ILC controller is used in the speed loop. The parameters of position loop's PI controller still remain the same. The velocity signal is also the signal which is gotten by the ESO. To satisfy the condition (18), The factor $\alpha$ and the deviation gain matrix $\Gamma$ are chosen as $\alpha=0.2$ and $\Gamma=0.5$. By the state space equation of system (14), it will have

$$
\begin{aligned}
\|(1-\alpha) I-\Gamma C B\| & =\left\|(1-0.2) I-0.5 \times\left[\begin{array}{ll}
0 & 1
\end{array}\right]\left[\begin{array}{l}
0 \\
1
\end{array}\right]\right\| \\
& =0.3<1 .
\end{aligned}
$$

The three controllers are tested for a sinusoidal signal, whose velocity and acceleration are $\omega=1.0\left({ }^{\circ} / \mathrm{s}\right)$ and $\alpha=$ $3.14\left(\mathrm{~s}^{\circ}\right)$. The period of $T$ sinusoidal signal is $2(\mathrm{~s})$.

The desired reference trajectory is shown in Figure 3. And the corresponding tracking performance under the three controllers is shown in Figures 4-6. As seen, the PI-PI controller has a larger tracking error whose maximum value is about $28.84^{\prime \prime}$. Comparatively speaking, the PI controller with ESO has a better tracking performance, which illustrates that ESO has an excellent estimation ability for disturbances involved in the system. Furthermore, the proposed PI-ILCESO controller has the least tracking error than other control methods, whose maximum value of error is about $20.06^{\prime \prime}$. This controller combines the advantages of ESO with the advantages of ILC. It makes use of ESO to eliminate most of the nonlinear disturbances. Simultaneously, the ILC gets the best control input by learning previous control information. Some detailed results of the three controllers are shown in Table 1.

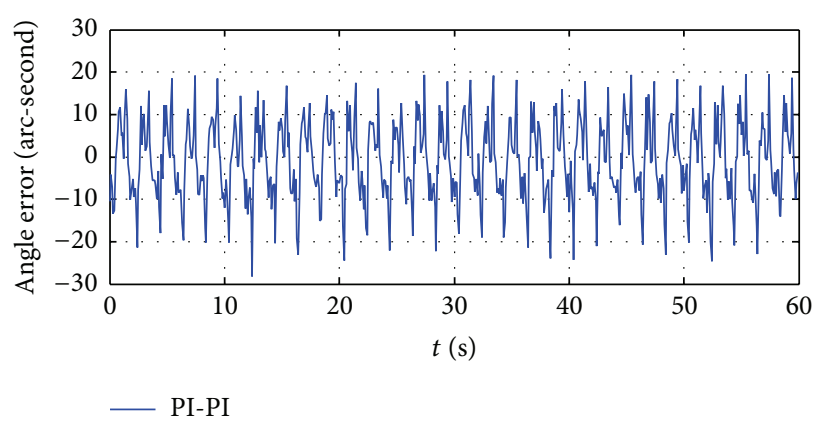

FIgURE 4: The tracking error of PI-PI.

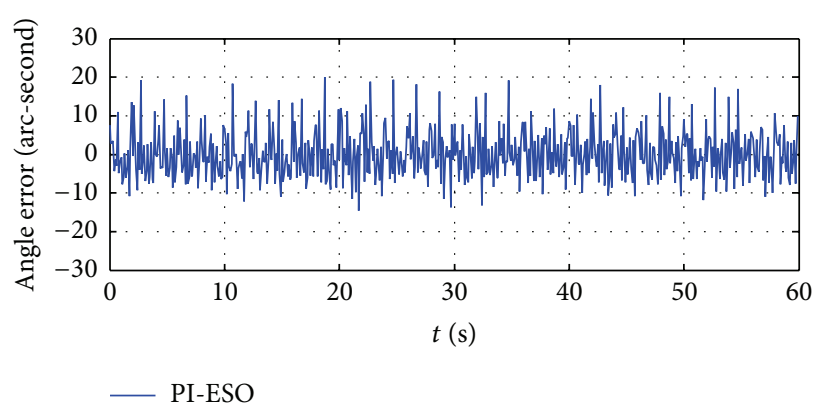

FIgURE 5: The tracking error of PI-ESO.

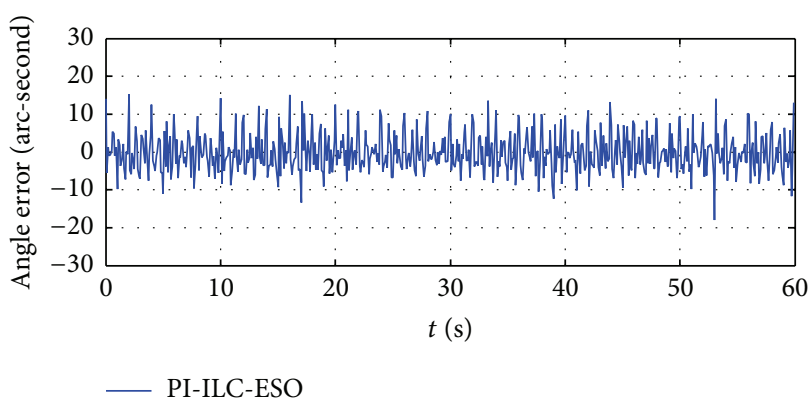

FIgURE 6: The tracking error of PI-ILC-ESO.

TABLE 1: The experimental results of the three controllers.

\begin{tabular}{lccc}
\hline & PI-PI & PI-ESO & PI-ILC-ESO \\
\hline |maximum error| & $28.8432^{\prime \prime}$ & $20.9988^{\prime \prime}$ & $20.0592^{\prime \prime}$ \\
Root mean square error & $9.8301^{\prime \prime}$ & $6.0282^{\prime \prime}$ & $5.6468^{\prime \prime}$ \\
\hline
\end{tabular}

From Table 1, it can be seen that the PI-ILC-ESO has better performance than other control methods. However, comparing the PI-ESO controller with the PI-ILC-ESO controller, it can be seen that the effectiveness of using ILC is not obvious. The maximum error reduces only to $0.94^{\prime \prime}$ and the root mean square error reduces to $0.38^{\prime \prime}$. In fact, Comparing with Figures 5 and 6, it can be found that the errors of many position points exceed $10^{\prime \prime}$ and the errors are close to $20^{\prime \prime}$, when the PI-ESO controller is used. But when the PI-ILC-ESO controller is used, almost all the errors of position points are near $10^{\prime \prime}$. So the conclusion that the comprehensive performance of PI-ILC-ESO is better than 
other control methods can be gotten. The ILC method helps the system get the best input signal. Besides, in Figures 5 and 6 , it can be found that the errors are evenly distributed. Comparing with the Figure 4 (PI-PI controller), both of the two controllers have reduced almost all the turning errors of system. The remaining errors are mostly caused by the signal noise. So it is difficult to reduce the remaining error and further improve the precision of system, unless the problem of signal noise is solved.

\section{Conclusion}

Uncertain nonlinear disturbances have been the major factors which restrict the performance of tracking control systems. This is because the systems will have a low tracking precision when some uncertain nonlinear disturbances are induced in the systems. Therefore, to reduce the influence introduced by the uncertain nonlinear disturbances, an ILC method with ESO is proposed in this paper. The ILC can get an excellent input signal by learning previous control information. It owns a better ability for eliminating some period disturbances. Meanwhile, an ESO is designed for estimating some uncertain nonlinear disturbances. It compensates the shortage of the ILC's disablement for nonperiod disturbances. In addition, the ESO can estimate an accurate velocity signal and supply the velocity as the feedback input signal of iterative learning controller. Furthermore the convergence analysis of the proposed control method guarantees the robustness of system. Finally, the experiment results show that the proposed control method has excellent performance for reducing the tracking error of telescope system.

\section{Conflict of Interests}

The authors declare that there is no conflict of interests regarding the publication of this paper.

\section{References}

[1] G. G. Valyavin, V. D. Bychkov, M. V. Yushkin et al., "Highresolution fiber-fed echelle spectrograph for the 6-m telescope. I. Optical scheme, arrangement, and control system," Astrophysical Bulletin, vol. 69, no. 2, pp. 224-239, 2014.

[2] K. Lu and Y. Xia, "Adaptive attitude tracking control for rigid spacecraft with finite-time convergence," Automatica, vol. 49, no. 12, pp. 3591-3599, 2013.

[3] Y.-J. Sun, "Composite tracking control for generalized practical synchronization of Duffing-Holmes systems with parameter mismatching, unknown external excitation, plant uncertainties, and uncertain deadzone nonlinearities," Abstract and Applied Analysis, vol. 2012, Article ID 640568, 11 pages, 2012.

[4] Y. Fu and T. Chai, "Self-tuning control with a filter and a neural compensator for a class of nonlinear systems," IEEE Transactions on Neural Networks and Learning Systems, vol. 24, no. 5, pp. 837-843, 2013.

[5] M. M. Bridges, D. M. Dawson, and J. Hu, "Adaptive control for a class of direct drive robot manipulators," International Journal of Adaptive Control and Signal Processing, vol. 10, no. 4-5, pp. 417-441, 1996.
[6] A. Tadayoni, W.-F. Xie, and B. W. Gordon, "Adaptive control of harmonic drive with parameter varying friction using structurally dynamic wavelet network," International Journal of Control, Automation and Systems, vol. 9, no. 1, pp. 50-59, 2011.

[7] Y. Alipouri and J. Poshtan, "Designing a robust minimum variance controller using discrete slide mode controller approach," ISA Transactions, vol. 52, no. 2, pp. 291-299, 2013.

[8] B.-Z. Guo and F.-F. Jin, "Sliding mode and active disturbance rejection control to stabilization of one-dimensional anti-stable wave equations subject to disturbance in boundary input," IEEE Transactions on Automatic Control, vol. 58, no. 5, pp. 1269-1274, 2013.

[9] Y.-S. Lu and S.-M. Lin, "Disturbance-observer-based adaptive feedforward cancellation of torque ripples in harmonic drive systems," Electrical Engineering, vol. 90, no. 2, pp. 95-106, 2007.

[10] T. Kavli, "Frequency domain synthesis of trajectory learning controllers for robot manipulators," Journal of Robotic Systems, vol. 9, no. 5, pp. 663-680, 1992.

[11] Y.-C. Wang and C.-J. Chien, "An observer-based adaptive iterative learning control using filtered-FNN design for robotic systems," Advances in Mechanical Engineering, vol. 6, Article ID 471418, 2014.

[12] S. Arimoto, S. Kawamura, and F. Miyazaki, "Bettering operation of Robots by learning," Journal of Robotic Systems, vol. 1, no. 2, pp. 123-140, 1984.

[13] J.-X. Xu, D. Huang, V. Venkataramanan, and The Cat Tuong Huynh, "Extreme precise motion tracking of piezoelectric positioning stage using sampled-data iterative learning control," IEEE Transactions on Control Systems Technology, vol. 21, no. 4, pp. 1432-1439, 2013.

[14] M. Norrlöf, "An adaptive iterative learning control algorithm with experiments on an industrial robot," IEEE Transactions on Robotics and Automation, vol. 18, no. 2, pp. 245-251, 2002.

[15] J.-X. Xu, Y. Chen, T. H. Lee, and S. Yamamoto, "Terminal iterative learning control with an application to RTPCVD thickness control," Automatica, vol. 35, no. 9, pp. 1535-1542, 1999.

[16] S. S. Saab, "A discrete-time stochastic learning control algorithm," IEEE Transactions on Automatic Control, vol. 46, no. 6, pp. 877-887, 2001.

[17] K. K. Leang and S. Devasia, "Design of hysteresis-compensating iterative learning control for piezo-positioners: application to atomic force microscopes," Mechatronics, vol. 16, no. 3-4, pp. 141-158, 2006.

[18] Z. Zhu, D. Xu, J. Liu, and Y. Xia, "Missile guidance law based on extended state observer," IEEE Transactions on Industrial Electronics, vol. 60, no. 12, pp. 5882-5891, 2013.

[19] M. Pizzocaro, D. Calonico, C. Calosso et al., "Active disturbance rejection control of temperature for ultrastable optical cavities," IEEE Transactions on Ultrasonics, Ferroelectrics, and Frequency Control, vol. 60, no. 2, pp. 273-280, 2013.

[20] J. Yao, Z. Jiao, and D. Ma, "Extended-state-observer-based output feedback nonlinear robust control of hydraulic systems with backstepping," IEEE Transactions on Industrial Electronics, vol. 61, no. 11, pp. 6285-6293, 2014.

[21] J. Yao, Z. Jiao, and D. Ma, "Adaptive robust control of dc motors with extended state observer," IEEE Transactions on Industrial Electronics, vol. 61, no. 7, pp. 3630-3637, 2014.

[22] Q. Zheng, L. Q. Gao, and Z. Gao, "On stability analysis of active disturbance rejection control for nonlinear time-varying plants with unknown dynamics," in Proceedings of the 46th IEEE 
Conference on Decision and Control (CDC '07), pp. 3501-3506, New Orleans, La, USA, December 2007.

[23] W. Qian, S. K. Panda, and J.-X. Xu, "Torque ripple minimization in PM synchronous motors using iterative learning control," IEEE Transactions on Power Electronics, vol. 19, no. 2, pp. 272279, 2004.

[24] G. Heinzinger, D. Fenwick, B. Paden, and F. Miyazaki, "Stability of learning control with disturbances and uncertain initial conditions," IEEE Transactions on Automatic Control, vol. 37, no. 1, pp. 110-114, 1992. 


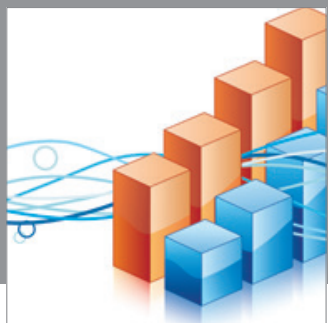

Advances in

Operations Research

mansans

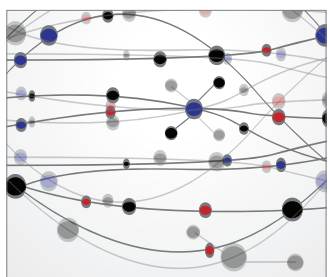

The Scientific World Journal
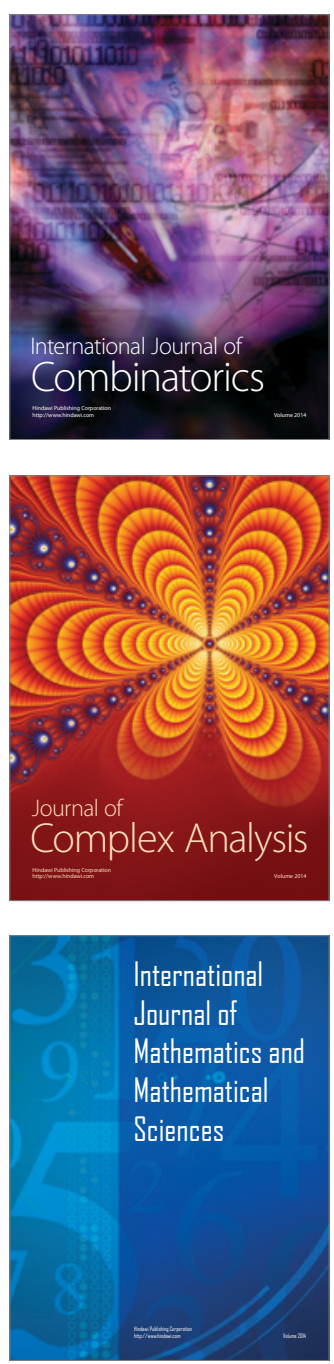
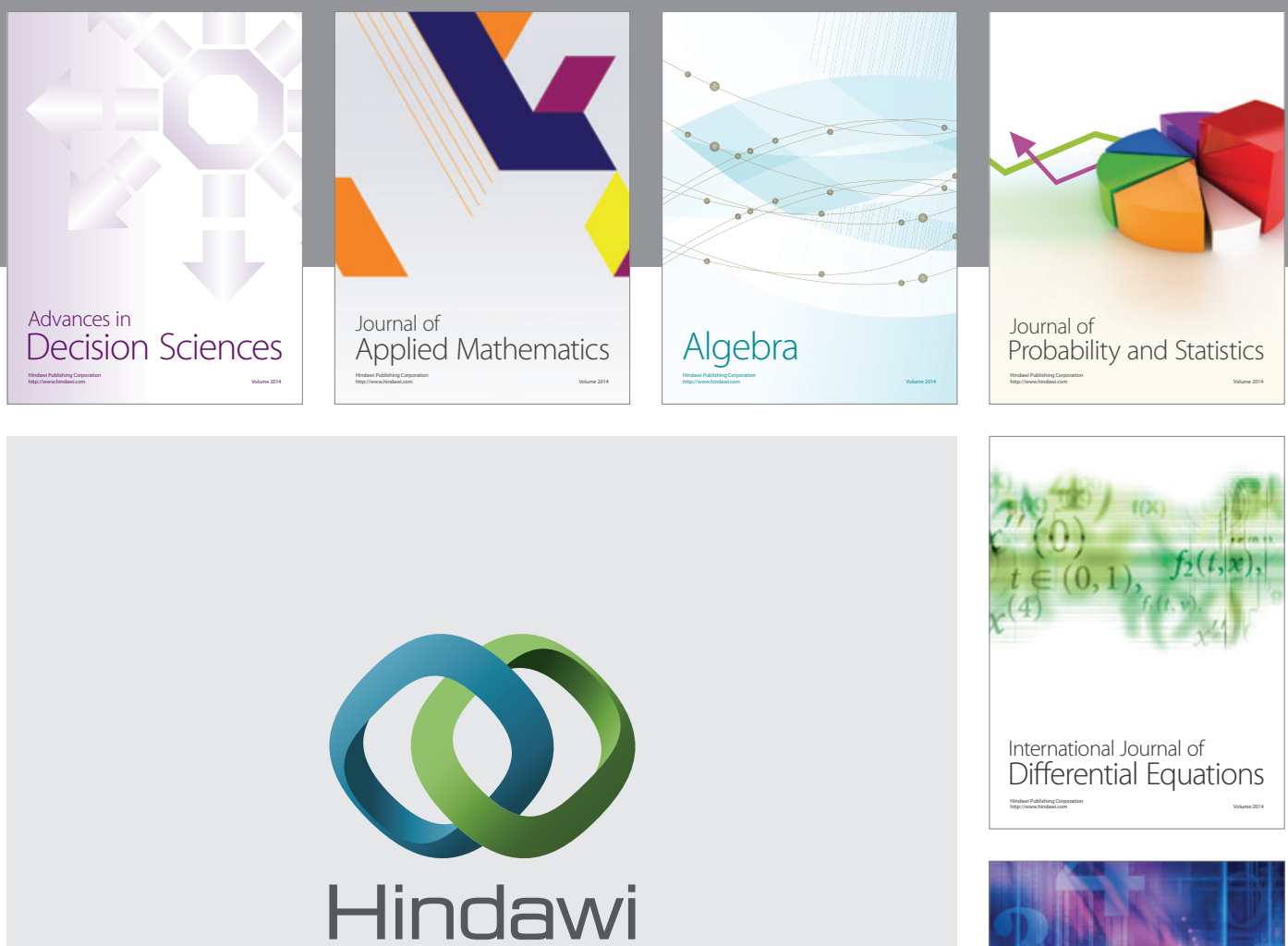

Submit your manuscripts at http://www.hindawi.com
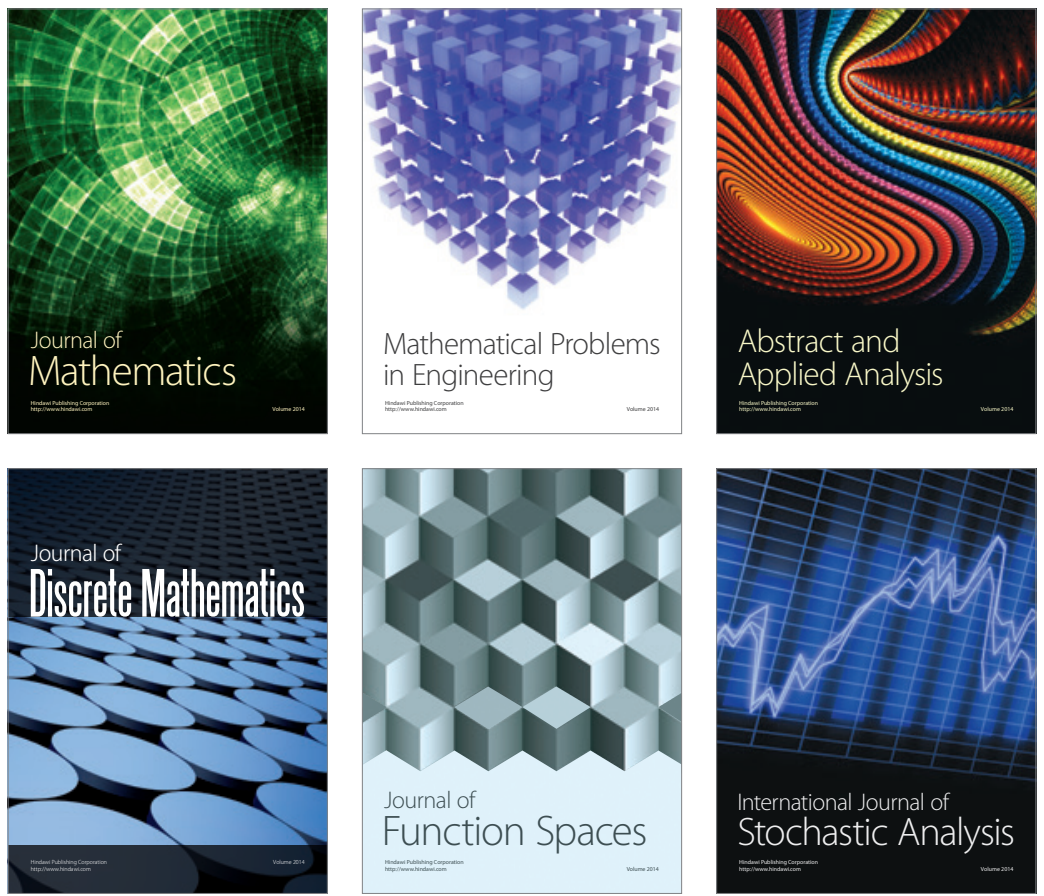

Journal of

Function Spaces

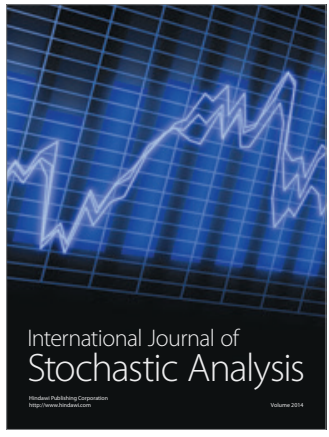

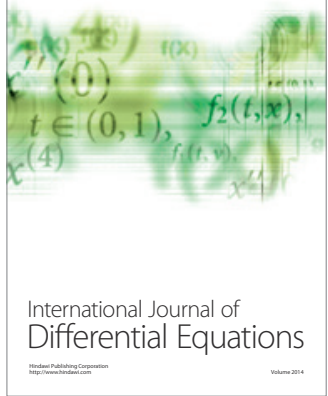
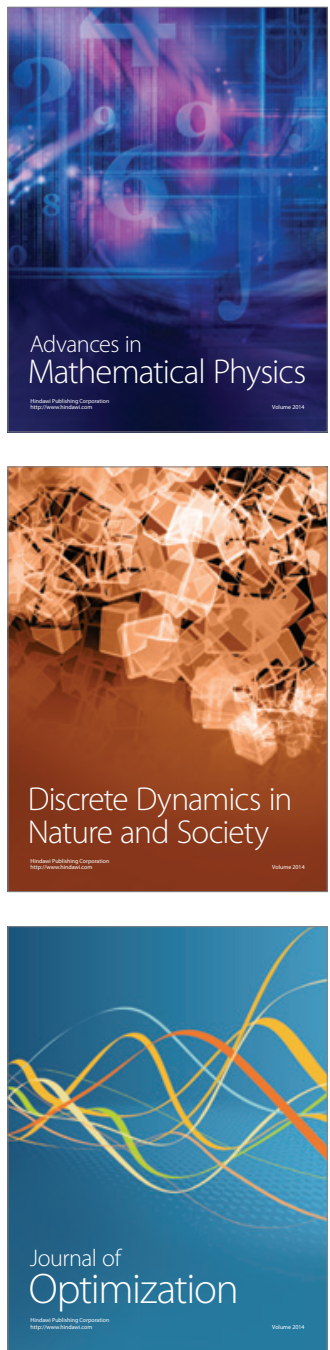